\title{
Review of: "Evidence for increased breakthrough rates of SARS-CoV-2 variants of concern in BNT162b2 mRNA vaccinated individuals"
}

Chandrasekhar Yadavalli ${ }^{1}$, Anil Mishra ${ }^{1}$

1 Tulane University

Potential competing interests: The author(s) declared that no potential competing interests exist.

\section{Commentary}

Increased concern rates of SARS-CoV-2 variants BNT162b2 in vaccinated individuals

Chandra Sekhar Yadavalli, PhD and Anil Mishra, PhD*

Tulane Eosinophilic Disorder Center, School of Medicine, Section of Pulmonary Diseases, 1430 Tulane Ave., SL-9, New Orleans, LA 70112-2699 * Corresponding author; email: amishra@tulane.edu

As the world is engaged in finding the vaccine for the SARS-CoV-2 but the CoV-2 has been changing its directly of its infection to the immune system in the form of variants of concern (VOC), which is an emergency topic must be addressed. Since its initial emergence, SARS-CoV-2 has undergone significant evolution, including the appearance of dominant variants with a D614G mutation15 (Wang et al., 2021). Viruses with this mutation alone, on the other hand, do not seem to be antigenically distinct. In this scenario, novel variants with mutations and deletions in the receptor-binding domain (RBD) and $\mathrm{N}$-terminal domain of $\mathrm{S}$ protein, as well as other proteins, have recently been discovered in few countries (Tegally et al., 2021). In the United Kingdom, the B.1.1.7 variant was discovered, in South Africa, the B.1.351 variant (also known as 501Y.V2) was discovered, and in Brazil, the P.1 and P.2 lineages were discovered (Davies et al., 2021a). SARS-CoV-2 B.1.1.7, also known as 501Y V1 in the GR clade was discovered in southeast England in September 2020 and quickly became the dominant variant in the UK, possibly due to its increased transmissibility 11 . This strain has now spread to over 50 countries, and there are indications that it may be more virulent than previously thought (Davies et al., 2021b). The B.1.1.7 contains eight mutations in the spike gene, including two deletions (H69/V70 and Y144) in the N-terminal domain (NTD), one substitution (N501Y) in the receptor-binding domain (RBD), and one substitution (P681H) near the furin cleavage site (Rambaut et al., 2020). SARS-CoV-2 B.1.351, also known as 501Y.V2 in the GH clade, was discovered in the Eastern Cape of South Africa in late 2020. Since then, this variant has become dominant locally, raising the possibility that it, too, has increased transmissibility. In addition to the D614G mutation, similarly the B.1.351 has nine mutations in the spike gene, including a cluster of mutations in the NTD, three substitutions (K417N, E484K, and N501Y) in the RBD, and one substitution (A701V) near the furin cleavage site (Tegally et al., 2020). In a recent reported studies a group of Israeli researchers,s published a review in the "medRxiv, a Cold spring Harbor Laboratory, BMJ and Yale group of online 
publication entitled "Evidence for increased breakthrough rates of SARS-CoV-2 variants of concern in BNT162b2 mRNA vaccinated individuals." These researchers emphasized the hypothesis that certain SARS-CoV-2 variants of concern can overcome protection bestowed by Pfizer-BioNTech (BNT162b2) mRNA vaccine and found confirmation of increased breakthrough rates in vaccinated individuals. In this study the researchers found a reduction in neutralization properties against the UK (B.1.1.7) and South African (B.1.351) variants. T-cell responses, which are not captured by neutralization studies, are also said to be stable after subsequent vaccination against these variants. As a result, it is unknown whether VOCs can achieve BNT162b2 vaccine breakthrough in real-world settings where the vaccine maintains both antibody and T-cell responses. However, the suitability of these assays in real-life circumstances is still unclear. The researchers identified symptomatic or asymptomatic individuals with confirmed SARS-CoV-2 infection among Clalit Health Services members, which is Israel's largest health-care agency. To minimize bias linked with pluralistic approach, each 'vaccine' was paired with an unvaccinated control with similar demographic characteristics.

The study was carried out initially with RNA extracted from polymerase chain reaction (PCR) samples and complete viral genome sequencing was performed. The viral sequence data was analyzed by using a strict lineage assignment technique for each viral sequence between the paired set of vaccinated and non-vaccinated patients. In this they found that only 433 patients (12.4\%) of the 3,491 infections detected among partially or fully vaccinated cases in the 4.7 million patients studied were able to be matched with a control and sequenced (not showed in the article, collected from internet). $92 \%$ of SARS-CoV-2 infections were detected within 28 days of the first vaccine dose in the partial effectiveness cases ( $\mathrm{n}$ $=247)$. Later one-third $(31 \%)$ were detected $21+$ days after the second vaccine dose in the full effectiveness cases $(n=$ 149). In vaccinated cases and unvaccinated controls, the B.1.1.7 variant was responsible for $90 \%$ of SARS-CoV-2 infections; only 11 infections of the B.1.351 variant was detected in case-control pairs. In a nutshell, the findings show an increased incidence of South African SARS-CoV-2 variant of concern in fully vaccinated individuals and an increased incidence of UK SARS-CoV-2 variant of concern in partially vaccinated individuals. More specifically, there was a disproportionally higher infection rate with the South African variant in 'vaccinees' infected at least one week after the second dose. Those infected between two weeks after the first dose and one week after the second dose, on the other hand, were disproportionately infected by the UK variant. This suggests that under different timing and dosage conditions, the vaccine's effectiveness against both variants of concern are reduced. However, the incidence of the South African variant in Israel remains low, and vaccine effectiveness against the UK strain remains high in fully vaccinated individuals. Overall, this is one of the first studies that evaluated the relative inclination for breakthrough infections between SARSCoV-2 lineages among BNT162b2 vaccine recipients in the observational scenario of a non-controlled patients. Taken together, the study was well organized with proper methods, experiments, analysis of data and finally the interpretation of the results was remarkable and supportive.

\section{References}

1. Wang, Pengfei, et al. "Antibody resistance of SARS-CoV-2 variants B. 1.351 and B. 1.1. 7." Nature (2021): 1-6.

2. Tegally, Houriiyah, et al. "Emergence of a SARS-CoV-2 variant of concern with mutations in spike glycoprotein." Nature (2021): 1-8.

3. Davies, Nicholas G., et al. "Estimated transmissibility and impact of SARS-CoV-2 lineage B. 1.1. 7 in England." Science 372.6538 (2021). 
4. Davies, Nicholas G., et al. "Increased hazard of death in community-tested cases of SARS-CoV-2 Variant of Concern 202012/01." MedRxiv (2021).

5. Tegally, Houriiyah, et al. "Emergence and rapid spread of a new severe acute respiratory syndrome-related coronavirus 2 (SARS-CoV-2) lineages with multiple spike mutations in South Africa." medRxiv (2020).

6. Rambaut, A., et al. "Preliminary genomic characterisation of an emergent SARS-CoV-2 lineage in the UK defined by a novel set of spike mutations." Genom. Epidemiol (2020). 